\title{
Blind spot in sepsis management - Tissue level changes in microcirculation
}

\author{
G. XANTUS ${ }^{1 *} \odot$, P. ALLEN ${ }^{2} \odot$ and P. KANIZSAI ${ }^{3}$ \\ ${ }^{1}$ School of Medicine, Cardiff University, Cardiff CF10 3AT, UK \\ ${ }^{2}$ Rural Clinical School, College of Health and Medicine, Burnie, Tasmania, 7320, Australia \\ ${ }^{3}$ Department of Emergency Medicine, Clinical Centre, University of Pécs, Pécs, Hungary
}

Received: July 23, 2020 • Accepted: January 20, 2021

Published online: April 9, 2021

(C) 2021 Akadémiai Kiadó, Budapest

\begin{abstract}
In sepsis cytokine-mediated inflammation, clotting cascade activation and glycocalyx shedding impair both function and structure of the microcirculation, compromising adequate tissue oxygenation/perfusion. Such mismatch results in "dysoxia", an imbalance in mitochondrial respiration.

Microvessel injuries can be grouped into four types: cytotoxic oedema, micro-vessel heterogeneity, sluggish/absent flow, and focal anaemia. Recognition of such diversity in microcirculatory pathology, alongside with the implementation of novel biomarkers might reveal previously unobserved heterogeneity in adults diagnosed with sepsis. Early identification of distinct subtypes may help not only to better stratify disease severity but may also provide explanation to the often seen insufficient/absent response to resuscitative treatment. Experimental evidence suggests that impaired microcirculatory flow may correlate with organ dysfunction and mortality. Therefore, reliable/reproducible diagnostic tools, that provide real-time information about the dynamic state of the microcirculation, might be practice changers in managing the critically ill.

The sublingual mucosa and the nailfolds provide easy access to microcirculation via hand-held, pointof-care devices. Accessing these windows, clinicians may recognise, understand and potentially correct the underlying tissue oxygenation/perfusion mismatch. This new clinical information might facilitate an individualised approach vs protocolised care aiming to administer the right balance of intravenous fluids/ vasopressors, time/dose auxiliary treatment modalities and, most importantly, might also guide determining the optimal duration of resuscitation to avoid/minimise harm and maximise benefits in sepsis management. However, before every-day clinical use of such point-of-care microcameras, validation studies are needed to establish not only feasibility but reliability and reproducibility as well.
\end{abstract}

\footnotetext{
* Corresponding author. University Emergency Department, Clinical Centre, University of Pécs, Hungary Ifjúság útja 13, Pécs, 7624, Hungary. Tel.: +44 7542236116. E-mail: gabor.xantus@gmail.com
} 


\section{INTRODUCTION}

For over 20 years sepsis resuscitation - restoration of tissue oxygenation/perfusion (TOP) - has traditionally been guided by indirect, often arbitrary parameters like lactate level/clearance [1], blood pressure, pulse pressure variations, central venous collapsibility index, etcetera. Both the SIRS based recognition/treatment and the new SOFA/qSOFA approach failed to significantly improve certain important patient-centred outcomes like one-year mortality, length of hospital stay, proportion of iatrogenic harm, etcetera, in adult sepsis. Interestingly, more are investigating the optimal timing/dose of sepsis recommendations [2,3]. Achievements must be reviewed critically; one might need to steer off the beaten track and look at sepsis management from a different perspective. The authors opine that the protocolised blanket approach in sepsis may be insufficient to recognise and correct potential underlying microcirculatory injuries.

In the early stages of sepsis, pro-inflammatory cytokines are released as part of the systemic immune response. Such cytokine-mediated inflammation, clotting cascade activation and, glycocalyx shedding impair both the functional and structural integrity of the microcirculation, the network of vessels that enables oxygen and substrate delivery at tissue level [4]. This complex system includes afferent arterioles, efferent venules, capillaries, and the microlymphatic network, all under $<100 \mu \mathrm{m}$ in diameter.

It also seems that in sepsis, nitric oxide (NO) might be one of the key players in the microcirculatory pathophysiology. During disease progression, in various end organs [5] the inducible NO-synthase enzyme is heterogeneously expressed resulting in the formation of pathologic arteriovenous shunts thus impeding adequate TOP. Microvascular flow might further be worsened by adverse structural changes like stiffening. Rigidity in the walls of the microvessels decrease vasodilator release capacity, impairing the flow of red blood cells. Respecting the diffuse, heterogenous functional/structural changes in TOP, to describe cellular hypoxia, and (focal) mitochondrial respiration insufficiency [6] a new definition of "dysoxia" was recommended [7]. The compromised microcirculatory circulation/blood flow seemingly correlates with organ dysfunction and mortality in the critically ill, hence a need arisen for diagnostic tests to assess mitochondrial-level "dysoxia" [8]. Nicotinamide adenine dinucleotide (NADH) fluorometry can identify tissue level dysoxia in-vitro; however, this method is not feasible in the clinical practice [6]. Therefore, bedside (point-of-care) tests, which can reliably inform clinicians in real-time about the dynamic state of the microcirculation might be a practice changer.

\section{The importance of microcirculation injuries in sepsis}

Real-time point-of-care sublingual microcirculation assessment enables the early recognition of both tissue level impairment in flow/circulation and the effect of fluid/vasopressor resuscitation to the microvessels in the oral mucosa [9] however, as yet the exact relationship between microcirculation injuries and either the end-organ failure or disease progression in adult sepsis is not completely understood [10]. Also, the observed association between the impaired sublingual microcirculation and disease severity does not imply linear prediction of impending organ failure.

Presently, numerous techniques are under evaluation to assess the quality and quantity of tissue level flow however, none has been implemented in the routine care. Sublingual microscopy [11], laser doppler assessment [12], glycocalyx measurements [13], Optical Coherence 
Tomography Angiography [14], nail-fold microscopy and Near Infrared Spectroscopy [15] all aim to provide useful parameters. Our paper is not intended to detail the individual assessment techniques but to raise awareness of the pathophysiological importance of the integrity of the microcirculation in adult sepsis.

Microvessel injuries (assessed by sublingual microcameras), can be categorised into 4 types: micro-vessel heterogeneity (T1), focal anaemia (T2), sluggish/absent flow (T3) and cytotoxic oedema (T4) [2]. The clinical use of the above subtypes might help to stratify septic patients and also might help to understand the absent or insufficient response to resuscitative efforts in the seemingly homogenous group of septic adults. Previous studies on microcirculation impairment in sepsis suggested a gap (uncoupling) between the major haemodynamic- and the tissue level microcirculation parameters. This phenomenon was defined as the "loss of haemodynamic coherence" [16]. Clinical studies demonstrated that in adult sepsis, despite acceptable macrocirculatory parameters (mean arterial pressure, central venous $\mathrm{O}^{2}$ saturation, Cardiac Index, etc.), microcirculation could still be severely compromised [17]. It also became evident that the impaired microcirculation in certain cases cannot necessarily be improved by increased flow of supplemental oxygen, vasopressor administration and/or rapid boluses of crystalloids (Fig. 1).

As a consequence of the dysregulated host response injures microvessels. Microcirculation injuries can be classified (Type 1-4) based on the mechanism explained below.

\section{Microcirculation assessment as a diagnostic aid}

Small-scale clinical studies in adult sepsis showed that survivors have better sublingual microcirculation parameters than non-survivors; the "proportion of perfused vessels", i.e., the ratio of
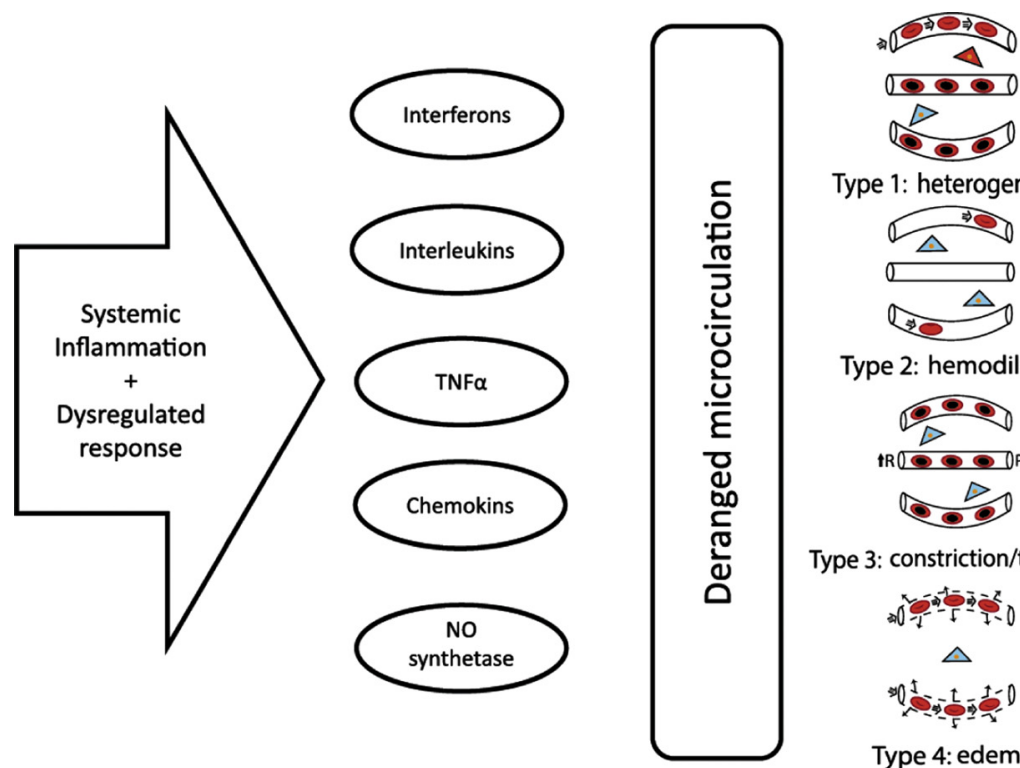

Type 1: heterogeneity

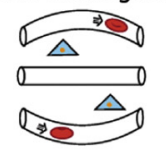

Type 2: hemodilution

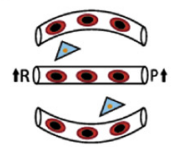

Type 3: constriction/tamponade

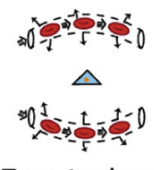

Type 4: edema

Fig. 1. Sepsis related pathophysiological changes in microcirculation 
perfused microvessels might be as strong an independent predictor of the outcome as lactate level [18]. However, low-level observational evidence is insufficient to pave the way from translational medicine to bedside; diagnostic/assessment tools in the first place must meet the Wilson and Jungner criteria for feasibility [19] and must repeatedly demonstrate high enough sensitivity and specificity together with excellent inter-rater agreement (QUADAS) at reasonable price to reliably aide management. Translational and clinical evidence suggests that the third generation sublingual microcameras may potentially both be feasible (see Table 1) and clinically reliable regardless if used by doctors or nurses [20]. Unfortunately, estimating parameters of microcirculatory flow still need a time-consuming offline evaluation or clinical semiquantitative scores based on trained observation. The latter was validated in management of haemorrhagic shock [21] however, in sepsis this has not yet been standardised hence, might be affected by sampling and spatial heterogeneity. Until further validation, assessment with microcameras can only provide association (not causation) between resuscitative measures and disease outcome/ progression.

Table 1. The summary of the Wilson Jungner criteria in relation to microcirculation assessment

\section{Principles}

The condition sought should be an important health problem.

There should be an accepted treatment for patients with recognized disease.

Facilities for diagnosis and treatment should be available.

There should be a recognizable latent or early symptomatic stage.

There should be a suitable test or examination.

The test should be acceptable to the population.

The natural history of the condition, including development from latent to declared disease, should be adequately understood.

There should be an agreed policy on whom to treat as patients.

The cost of case finding (including diagnosis) should be economically balanced in relation to possible expenditure on medical care as a whole.

Case finding should be a continuing process and not a "once and for all" project.
Sublingual microcirculation assessment in sepsis

Sepsis has high mortality and poses a significant economic burden to hospitals.

Well described protocols and treatment bundles are available.

Sepsis recognition and management is well established in prehospital and hospital settings alike. Sepsis severity is well stratified.

Lactate levels ScrO2, SVV, PCT values are used in setting up diagnosis and management plans.

Sublingual microcirculation assessment in sepsis is tried and published in Europe, the UK and the USA. Sepsis is subject of continuous research; this method might be a useful adjunct.

Surviving Sepsis Campaign, BUFALO, Sepsis-6.

The treatment of sepsis is very cost intensive. Any initiative to reduce cost might have huge bearing on financing.

Sublingual microcirculation assessment can be used in both ICU and emergency settings, as the device is reasonably priced, the maintenance and the education costs are low. 
Box 1. Peripheral oxygen delivery can be described by the below equation.

$$
\mathrm{DO}_{2}=\mathrm{HR} \times \mathrm{SV} \times\left(1.39 \times \mathrm{Hb} \times \mathrm{O}_{2} \mathrm{Sat} / 100\right)+\left(0.003 \times \mathrm{PO}_{2}\right)
$$

$\mathrm{DO}_{2}=$ peripheral oxygen delivery, $\mathrm{HR}=$ heart rate, $\mathrm{SV}=$ stroke volume, $\mathrm{Hb}=$ haemoglobin, $\mathrm{O}_{2}$ Sat is the tissue level saturation, $\mathrm{PO}_{2}=$ partial tissue oxygen pressure.

\section{A hypothetical basis how microcirculation assessment may aid resuscitation in sepsis}

Physicians treating septic adults must fully understand the complexity of the peripheral oxygen delivery and apply this knowledge in practice during resuscitation. To correctly treat the underlying tissue oxygenation/perfusion mismatch one must keep in mind the below formula (see Box 1).

The present emergency medicine approach (both the SIRS and the SOFA based) concentrates mainly on the correction of macrohemodynamic variables like systolic blood pressure (however, stroke volume has a nonlinear relation with the left ventricular pressure-volume), heart rate (which is often affected by comorbidities, concomitant medications, cardiotoxins, etc.), the peripheral oxygen saturation (which has a non-linear relationship with end-organ tissue oxygen saturation) hence inherently missing tissue level pathology. Such one-size-fits-all sepsis protocols have an apparent blind spot for tissue level drop in haemoglobin, saturation, partial pressures, which are probably equally (or more) important factors in correcting the peripheral perfusion than heart and respiration rate and systolic blood pressure. The authors opine, that a paradigm change might be needed, which would consider the correction of both micro-and macrocirculation pathology alike.

In theory, bedside assessment of the microcirculation (if meeting all the Wilson-Jungner and QUADAS criteria) would probably usefully complement the present monitoring strategy in sepsis. As microvascular injuries may provide insight into the exact nature of TOP, such knowledge might serve as a hypothetical base helping to balance crystalloids, vasopressors and other auxillary treatment options during resuscitation to correct (not only macrohemodinamic parameters but) the underlying tissue level pathology as well.

In the past decades, numerous "silver bullet" concepts have risen and fallen in sepsis. Colloids [22], activated protein-C [23], nitroglycerin [24], vitamin C [25], Na-acetyl-cysteine (NAC) [26], steroids [27], etc. seemed efficient in small-scale observations but they all failed to prevail in randomised control trials or in unbiased systematic reviews. Due to the inability to assess microcirculation and recognise the type of underlying TOP mismatch, even randomised studies (of inadequate power and/or if analysed with per protocol analysis) might have concealed certain unaccounted confounders. In theory, factoring tissue level microvascular injuries in the trial models might smoothen the incongruencies between the small-scale observational and major clinical trials in adult sepsis. Further clinical studies of high rigour are needed to determine the relationship between the above modalities and microcirculation injuries in adult sepsis.

In case of (cytotoxic) oedema due to endothelial damage and/or glycocalyx shedding, repeated fluid boluses are unlikely to improve tissue level perfusion/oxygenation irrespective of the cause (sepsis-related oxidative stress, endotoxin exposure or shearing forces of aggressive crystalloid resuscitation). When the barrier function of the capillaries has already been damaged, 
unnecessary further fluid replacement might worsen the "leak" and increasing the intercellular free water. The oedema would inevitably impair tissue hypoxia, because the oxygen diffusion distance increases and at the same time the dissolved oxygen concentration decreases (diffusion oxygen deficiency). In the recent years (recognising of the above), the potential iatrogenic harm of aggressive fluid resuscitation in sepsis has repeatedly been investigated [28].

Strictly on a hypothetical base, if sublingual microcirculation assessment confirms tissue level oedema (T4 injury), early administration of vasopressors might probably be more appropriate by sealing the leak and increasing the stroke volume. By repeated use of this non-invasive monitoring tool, the effect of the resuscitative efforts can theoretically be checked in real time. Such feed-back might be helpful to determine the dose/rate of vasopressors and crystalloids (titrate to effect) to maximise benefit and to minimise harm. While endothelial glycocalyx has long been identified to regulate vascular permeability (with subsequent oedema formation) [29, 30], at present neither the prognostic value of glycocalyx dimensions (measured sublingually in vivo) or microcirculation flow/perfusion parameters on organ damage in sepsis have been evaluated in large multi-centre studies. The available low-level observational evidence is seemingly equivocal: discordant changes were reported in association with disease progression/ outcome in sepsis [31].

In T1 injury (micro vessel heterogeneity) some capillaries are open; others are closed due to malfunctioning shunts. In these cases, vitamin C and/or NAC might probably be useful to correct the pathologic shunts and even up heterogeneity of the perfused vessels. In T3 injury (absent/sluggish flow) cautious administration of colloids, protein-C or inodilators might be helpful to open the microcirculation, increase red blood cell velocity and tissue perfusion. Conversely, in T3 injury excessive use of vasopressors might cause harm by further narrowing the afferent arterioles resulting in detrimental ischaemic injuries in vital organs like gut mucosa, renal parenchyma, etcetera [32]. T2 injuries (focal, dilutional anaemia) can be temporary or sustained, in this case the oxygen transport capacity is decreased because of low haemoglobin concentration resulting in convective oxygen deficiency. In these cases, the administration of red blood cells might increase the success of resuscitation. Obviously, in case of focal anaemia both excessive crystalloid boluses and/or vasopressors/inodilators might worsen TOP.

Current understanding of the association between the microcirculation characteristics and the outcome in sepsis is insufficient to prove or disprove the above theory. Even though microcirculation assessment has provided important observations in sepsis management; however, further pragmatic validation must determine if these novel equipment/concepts could safely be used in the everyday clinical practice in the Emergency Department.

\section{CONCLUSION}

Despite over two decades of improved awareness, relentless clinical efforts and a recent facelift in recognition/management, adult sepsis is still of high disease burden and mortality, which calls for a paradigm change. Individually targeted resuscitation might be needed rather than on-sizefits-all protocols. In adult sepsis, without bedside monitoring of the microcirculation, attending physicians may have neither sufficient data nor feedback on the appropriateness/success of their resuscitative efforts. Among other translational diagnostic adjuncts, sublingual microcameras might probably be useful to complement the macro-haemodynamic parameters-based 
assessment and aid management. Further clinical studies are needed to determine if point-ofcare microcirculation assessment may be a feasible and safe method to help finding the right balance between intravenous fluid replacement/vasopressors, the optimal timing/dosing of auxiliary treatment options and most importantly, if it might safely guide on the optimal duration of resuscitation to avoid and minimise harm, and to maximise treatment benefit.

\section{REFERENCES}

1. Xantus G, Kiss B, Molnar G, Matheson C, Anna Gyarmathy V, Kanizsai PL. Lactate reloaded-reevaluation of the importance of lactate monitoring in the management of adult sepsis in the emergency department. Biocell 2021; 45(3): 445-9. https://doi.org/10.32604/biocell.2021.014754.

2. Xantus G, Allen P, Norman S, Kanizsai P. Antibiotics administered within 1 hour to adult emergency department patients screened positive for sepsis: a systematic review. Eur J Emerg Med 2020 Aug; 27(4): 260-7. https://doi.org/10.1097/MEJ.0000000000000654. PMID: 31855885.

3. Macdonald SPJ, Keijzers G, Taylor DM, Kinnear F, Arendts G, Fatovich DM, et al. Restricted fluid resuscitation in suspected sepsis associated hypotension (REFRESH): a pilot randomised controlled trial. Intensive Care Med 2018 Dec; 44(12): 2070-8. https://doi.org/10.1007/s00134-018-5433-0. PMID: 30382308.

4. Guven G, Hilty MP, Ince C. Microcirculation: physiology, pathophysiology, and clinical application. Blood Purif 2020; 49(1-2): 143-50. https://doi.org/10.1159/000503775. PMID: 31851980; PMCID: PMC7114900.

5. Winkler MS, Kluge S, Holzmann M, Moritz E, Robbe L, Bauer A, et al. Markers of nitric oxide are associated with sepsis severity: an observational study. Crit Care 2017 Jul 15; 21(1): 189. https://doi.org/10.1186/s13054017-1782-2. PMID: 28709458; PMCID: PMC5513203.

6. Ince C, Mik EG. Microcirculatory and mitochondrial hypoxia in sepsis, shock, and resuscitation. J Appl Physiol (1985) 2016 Jan 15; 120(2): 226-35. https://doi.org/10.1152/japplphysiol.00298.2015. PMID: 26066826.

7. Creery D, Fraser DD. Tissue dysoxia in sepsis: getting to know the mitochondrion. Crit Care Med 2002 Feb; 30(2): 483-4. https://doi.org/10.1097/00003246-200202000-00036. PMID: 11889335.

8. Ince C, van der Sluijs JP, Sinaasappel M, Avontuur JA, Coremans JM, Bruining HA. Intestinal ischemia during hypoxia and experimental sepsis as observed by $\mathrm{NADH}$ videofluorimetry and quenching of Pdporphine phosphorescence. Adv Exp Med Biol 1994; 361:105-10. https://doi.org/10.1007/978-1-4615-18754_14. PMID: 7597932.

9. De Backer D, Orbegozo Cortes D, Donadello K, Vincent JL. Pathophysiology of microcirculatory dysfunction and the pathogenesis of septic shock. Virulence 2014 Jan 1; 5(1): 73-9. https://doi.org/10.4161/viru.26482. PMID: 24067428; PMCID: PMC3916386.

10. Edul VS, Ince C, Vazquez AR, Rubatto PN, Espinoza ED, Welsh S, et al. Similar microcirculatory alterations in patients with normodynamic and hyperdynamic septic shock. Ann Am Thorac Soc 2016 Feb; 13(2): 240-7. https://doi.org/10.1513/AnnalsATS.201509-606OC. PMID: 26624559.

11. Dilken O, Ergin B, Ince C. Assessment of sublingual microcirculation in critically ill patients: consensus and debate. Ann Transl Med 2020 Jun; 8(12): 793. https://doi.org/10.21037/atm.2020.03.222. PMID: 32647718; PMCID: PMC7333125.

12. Charlton M, Sims M, Coats T, Thompson JP. The microcirculation and its measurement in sepsis. J Intensive Care Soc 2017 Aug; 18(3): 221-7. https://doi.org/10.1177/1751143716678638. PMID: 29118834; PMCID: PMC5665123. 
13. Rovas A, Seidel LM, Vink H, Pohlkötter T, Pavenstädt H, Ertmer C, et al. Association of sublingual microcirculation parameters and endothelial glycocalyx dimensions in resuscitated sepsis. Crit Care 2019 Jul 24; 23(1): 260. https://doi.org/10.1186/s13054-019-2542-2. PMID: 31340868; PMCID: PMC6657098.

14. Hessler M, Nelis P, Ertmer C, Alnawaiseh M, Lehmann F, Schmidt C, et al. Author Correction: Optical coherence tomography angiography as a novel approach to contactless evaluation of sublingual microcirculation: a proof of principle study. Sci Rep 2020 Jul 7; 10(1): 11458. https://doi.org/10.1038/s41598-02068556-4. Erratum for: Sci Rep. 2020 Mar 25;10(1):5408. PMID: 32632131; PMCID: PMC7338536.

15. Barcelos A, Lamas C, Tibiriça E. Evaluation of microvascular endothelial function in patients with infective endocarditis using laser speckle contrast imaging and skin video-capillaroscopy: research proposal of a case control prospective study. BMC Res Notes 2017 Jul 28; 10(1): 342. https://doi.org/10.1186/s13104-017-26603. PMID: 28754178; PMCID: PMC5534027.

16. Hernandez G, Bruhn A, Luengo C, Regueira T, Kattan E, Fuentealba A, et al. Effects of dobutamine on systemic, regional and microcirculatory perfusion parameters in septic shock: a randomized, placebocontrolled, double-blind, crossover study. Intensive Care Med 2013 Aug; 39(8): 1435-43. https://doi.org/10. 1007/s00134-013-2982-0. PMID: 23740284.

17. Hernandez G, Luengo C, Bruhn A, Kattan E, Friedman G, Ospina-Tascon GA, et al. When to stop septic shock resuscitation: clues from a dynamic perfusion monitoring. Ann Intensive Care 2014 Oct 11; 4:30. https://doi.org/10.1186/s13613-014-0030-z. PMID: 25593746; PMCID: PMC4273696.

18. Kanoore Edul VS, Dubin A, Ince C. The microcirculation as a therapeutic target in the treatment of sepsis and shock. Semin Respir Crit Care Med 2011 Oct; 32(5): 558-68. https://doi.org/10.1055/s-0031-1287864. PMID: 21989692.

19. Andermann A, Blancquaert I, Beauchamp S, Déry V. Revisiting Wilson and Jungner in the genomic age: a review of screening criteria over the past 40 years. Bull World Health Organ 2008 Apr; 86(4): 317-9. https:// doi.org/10.2471/blt.07.050112. PMID: 18438522; PMCID: PMC2647421.

20. Tanaka S, Harrois A, Nicolaï C, Flores M, Hamada S, Vicaut E, et al. Qualitative real-time analysis by nurses of sublingual microcirculation in intensive care unit: the MICRONURSE study. Crit Care 2015 Nov 6; 19:388. https://doi.org/10.1186/s13054-015-1106-3. PMID: 26542952; PMCID: PMC4635966.

21. Hutchings SD, Naumann DN, Hopkins P, Mellis C, Riozzi P, Sartini S, et al. Microcirculatory impairment is associated with multiple organ dysfunction following traumatic hemorrhagic shock: the MICROSHOCK study. Crit Care Med 2018 Sep; 46(9): e889-96. https://doi.org/10.1097/CCM.0000000000003275. PMID: 29957708.

22. Rochwerg B, Alhazzani W, Sindi A, Heels-Ansdell D, Thabane L, Fox-Robichaud A, et al. Fluids in Sepsis and Septic Shock Group. Fluid resuscitation in sepsis: a systematic review and network meta-analysis. Ann Intern Med 2014 Sep 2; 161(5): 347-55. https://doi.org/10.7326/M14-0178. PMID: 25047428.

23. Zhang $Z$. The efficacy of activated protein $C$ for the treatment of sepsis: incorporating observational evidence with a Bayesian approach. BMJ Open 2015 Jan 16; 5(1): e006524. https://doi.org/10.1136/bmjopen-2014006524. PMID: 25596198; PMCID: PMC4298096.

24. Zhang Z, Chen K. Vasoactive agents for the treatment of sepsis. Ann Transl Med 2016 Sep; 4(17): 333. https:// doi.org/10.21037/atm.2016.08.58. PMID: 27713891; PMCID: PMC5050188.

25. Carr AC. Vitamin C administration in the critically ill: a summary of recent meta-analyses. Crit Care 2019 Jul 30; 23(1): 265. https://doi.org/10.1186/s13054-019-2538-y. PMID: 31362775; PMCID: PMC6664573.

26. Szakmany T, Hauser B, Radermacher P. N-acetylcysteine for sepsis and systemic inflammatory response in adults. Cochrane Database Syst Rev 2012 Sep 12; 2012(9): CD006616. https://doi.org/10.1002/14651858. CD006616.pub2. PMID: 22972094; PMCID: PMC6517277. 
27. Rochwerg B, Oczkowski SJ, Siemieniuk RAC, Agoritsas T, Belley-Cote E, D'Aragon F, et al. Corticosteroids in sepsis: an updated systematic review and meta-analysis. Crit Care Med 2018 Sep; 46(9): 1411-20. https://doi. org/10.1097/CCM.0000000000003262. PMID: 29979221.

28. Xantus G, Penny A, Norman SE, Kanizsai PL. A korai krisztalloidbolus elönyei felnőtt szeptikus betegek sürgösségi kezelésében [The role of early intravenous crystalloid bolus in adult sepsis resuscitation in the emergency department]. Orv Hetil 2020 Sep 27; 161(39): 1668-74. Hungarian. https://doi.org/10.1556/650. 2020.31864. PMID: 32980822.

29. Chelazzi C, Villa G, Mancinelli P, De Gaudio AR, Adembri C. Glycocalyx and sepsis-induced alterations in vascular permeability. Crit Care 2015 Jan 28; 19(1): 26. https://doi.org/10.1186/s13054-015-0741-z. PMID: 25887223; PMCID: PMC4308932.

30. Uchimido R, Schmidt EP, Shapiro NI. The glycocalyx: a novel diagnostic and therapeutic target in sepsis. Crit Care 2019 Jan 17; 23(1): 16. https://doi.org/10.1186/s13054-018-2292-6. PMID: 30654825; PMCID: PMC6337861.

31. Lee DH, Dane MJ, van den Berg BM, Boels MG, van Teeffelen JW, de Mutsert R, et al. NEO study group. Deeper penetration of erythrocytes into the endothelial glycocalyx is associated with impaired microvascular perfusion. PLoS One 2014 May 9; 9(5): e96477. https://doi.org/10.1371/journal.pone.0096477. PMID: 24816787; PMCID: PMC4015985.

32. Chen YY, Wu VC, Huang WC, Yeh YC, Wu MS, Huang CC, et al. Norepinephrine administration is associated with higher mortality in dialysis requiring acute kidney injury patients with septic shock. J Clin Med 2018 Sep 12; 7(9): 274. https://doi.org/10.3390/jcm7090274. PMID: 30213107; PMCID: PMC6162856. 